\title{
Les acteurs octogénaires et nonagénaires dans le théâtre polonais contemporain
}

Octogenarian and Nonagenarian Actors in Contemporary Polish Theater

Dorota Jarząbek-Wasyl

\section{OpenEdition}

\section{Journals}

Édition électronique

URL : https://journals.openedition.org/recherchestravaux/743

DOI : 10.4000/recherchestravaux.743

ISSN : 1969-6434

Éditeur

UGA Éditions/Université Grenoble Alpes

\section{Édition imprimée}

Date de publication : 28 mai 2015

Pagination : 107-120

ISBN : 978-2-84310-298-1

ISSN : 0151-1874

Référence électronique

Dorota Jarząbek-Wasyl, « Les acteurs octogénaires et nonagénaires dans le théâtre polonais contemporain », Recherches \& Travaux [En ligne], 86 | 2015, mis en ligne le 28 novembre 2016, consulté le 29 octobre 2021. URL : http://journals.openedition.org/recherchestravaux/743 ; DOI : https:// doi.org/10.4000/recherchestravaux.743 
Dorota JARZĄBEK-WASYL

Université Jagellone de Cracovie

\section{Les acteurs octogénaires et nonagénaires dans le théâtre polonais contemporain ${ }^{I}$}

\section{À la source du motif}

Dans la culture et la tradition théâtrale polonaises, le drame de Stanisław Wyspiański intitulé Affranchissement [Wyzwolenie, 1903] est une référence primordiale en ce qui concerne le topos du "vieil acteur». Les héros de la pièce sont des professionnels du monde du théâtre : le Poète, le Metteur en scène, la Muse (la première actrice), les techniciens. Dans le troisième acte, intervient le Vieil Acteur, qui fait la confidence suivante :

Jouer ne me fait pas vivre.

De plus personne ne se soucie de moi et on me regarde de travers.

Je ne m’en inquiète guère. - Mon temps est passé. Je n'espère plus rien.

Je me suis habitué à ces planches. Je peux m’en aller. - Je m’écarte.

J'ai poursuivi autrefois la gloire, je jouais Hamlet.

Aujourd'hui il y a d'autres Hamlet. - Une maison. - Des enfants. - Une femme. La gloire des artistes! Je ne m'étonne pas de ces lauriers.

J'en ai reçu un bon nombre, j'en avais les mains pleines,

Quand j'ai fêté le jour de mes trente ans de scène.

J'ai connu les applaudissements, la reconnaissance et la considération

Éphémères, comme la lumière d'une seule soirée,

qui s'éteint quand les rangées se vident.

[...]

Mon père était un héros, et moi je ne suis rien.

I. Traduction : Christophe Cadoux. L'article est une version étendue du texte publié dans les actes du colloque polonais-allemand Portret aktora - Ein Schauspielerporträt, A. R. Burzyńska (éd.), Szczecin, Biblioteka Kontrapunktu, 2013. 
Le rôle de bouffon était bien réussi, la comédie était ma vie,

La honte me fait rougir, me gêne, elle me couvre le visage...

Mon père était un héros, et nous, nous ne sommes rien $^{2}$ !

Ce monologue a sa légende. Le personnage du Vieil Acteur était en effet une personnalité historique. Wyspiański fait ici le portrait d'un de ses amis, un acteur de longue date de la scène cracovienne, Leon Stępowski (I852-I9I4). Stępowski venait d'une famille noble, son père avait participé à l'insurrection polonaise de janvier I863, pendant laquelle il perdit toute sa fortune. Contre la volonté de sa famille, Leon fit du théâtre. Au début, il jouait sous un pseudonyme, afin de ne pas entacher son nom de famille. Bien qu'il lui soit arrivé de jouer Hamlet - en province - il n'a cependant jamais fait une grande carrière artistique. Il connut pourtant toutes les splendeurs et misères d'un comédien du XIX ${ }^{\mathrm{e}}$ siècle ${ }^{3}$. Ce n'était pas un acteur d'exception, mais il avait du métier et il était consciencieux, travailleur et instruit. Il jouait «les utilités», comme on disait alors. Il s'adapta à tous les types de rôles, mais il obtint rarement un rôle de premier plan. Stępowski fut engagé au théâtre de Cracovie, au moment où y régnait l'école d'esthétique réaliste, dirigée par Stanisław Koźmian, l'équivalent polonais de l'Allemand Heinrich Laube. La formation professionnelle et culturelle qu'il y reçut influença toute sa carrière. Il était incapable de jouer autrement et ne voulait pas suivre des règles dérogeant à ses idéaux de jeunesse. Après des années de travail, il avait lui-même remarqué avec une certaine mélancolie son inadaptation à la nouvelle esthétique, étant mal à l'aise avec le répertoire moderne et le nouveau style de jeu. La meilleure preuve pour illustrer cela, c'est qu'en 1902, pour le trentième anniversaire de sa carrière, Stępowski choisit de jouer un rôle complètement démodé : celui de Michonnet dans Adrienne Lecouvreur.

Il est intéressant de remarquer que Stępowski, selon nos critères, n'était pas encore, en ce temps-là, un vieil homme. Au moment du jubilé, il avait tout juste cinquante ans. Mais, pour un acteur, plus que l'âge réel, c'était le temps passé sur les planches qui comptait, surtout à cette époque, où pratiquement tous les acteurs devaient endurer les conditions éreintantes et instables du théâtre itinérant. Leon Stępowski «se sentait vieux» pour d'autres raisons encore - il appartenait au type spécifique du puer senex. Dans sa jeunesse, il joua les pères et les vieillards et, finalement, devenu un homme d'âge mûr, ce

2. S. Wyspiański, Wyzwolenie [Affranchissement], dans S. Wyspiański, Dzieta zebrane [CEuvres choisies], éd. L. Płoszewski, Cracovie, Wydawnictwo Literackie, I959, vol. V, p. 176-I77 (en polonais; trad. C. Cadoux).

3. Voir D. Jarząbek-Wasyl, «Stary Aktor z Wyzwolenia. Próba portretu Leona Stępowskiego» [Le Vieil Acteur d'Affranchissement. Essai sur Leon Stępowski], Pamiętnik Teatralny, cahier I-2, 20I4, p. I53-I83; voir aussi : R. Włodek, «Stępowski Leon», Polski Stownik Biograficzny, vol. XLIII, cahier 179, Wrocław, 2005, p. 562-564. 
type de rôles coïncida avec sa vraie personnalité. Une certaine aura d'acteurphilosophe émanait naturellement de lui : un grand savoir, une conscience de soi, une gravité solennelle, une curiosité, mais aussi un discret sentiment d'aliénation ${ }^{4}$. D'un point de vue mental, spirituel et artistique, il appartenait à une époque révolue. Au cours de son jubilé, il surprit l'assemblée en recourant à une formule archaïque d'adresse à l'auditoire : «Majestueux Public de la Cité Royale de Cracovies». Après la célébration et les congratulations du jubilé, l'acteur rentra chez lui et, dans le cercle plus restreint de ses amis intimes et de sa famille, il donna une péroraison, qui inspira à Wyspiański le monologue du Vieil Acteur. Voilà, résumé en quelques mots, la préhistoire de cette tirade de L'Affranchissement. Par le truchement de Wyspiański, cette péroraison a acquis une plus grande signification, touchant à la structure archétypale de l'art et au métier d'acteur dans sa dimension existentielle.

Wyspiański a tiré une tragique conclusion des facteurs artistiques et personnels qui constituent la condition de l'homme de théâtre. Il a montré que le coût à payer pour ceux qui exercent cette profession réside dans la vie elle-même, avec toute l'intensité et la dépense d'énergie qu'elle suppose. Cette idée est exprimée par les phrases détachées et elliptiques du monologue du Vieil Acteur, qui débutent sur une syllepse de sens : "Jouer ne me fait pas vivre». Le verbe «vivre» renvoie ici autant à la subsistance qu'à l'existence. Quand l'acteur reçoit moins de rôles à jouer, ses facultés créatrices et sa consistance existentielle se réduisent, tout autant d'ailleurs que le sens qu'il a subjectivement donné à son existence. Être évincé de la scène est dès lors interprété comme non-existence.

"Je ne m'en inquiète guère. - Mon temps est passé. Je n'espère plus rien. I Je suis habitué à ces planches. Je peux m'en aller. - Je m'écarte.» Le vieil acteur approche de son heure de repos, mais il n'est pas définitivement libéré de l'influence de la scène. Il revient toujours dans son théâtre, et son attachement pour la scène est une question de coutumes propres au milieu artistique, en particulier au XIX ${ }^{e}$ siècle. Selon la tradition, les artistes usés avaient la possibilité de continuer à travailler dans le milieu du théâtre. Si, en raison de l'âge, de la maladie, de la perte de mémoire ou simplement du changement de répertoire, l'acteur ne pouvait plus se produire sur scène et qu'il n'avait aucune autre ressource pour vivre, il avait alors la possibilité de rester au théâtre et d'y être employé comme technicien. Les régisseurs, les souffleurs, les copistes, les employés du vestiaire avaient été auparavant acteurs. De même, les artistes qui

4. A. Grzymała-Siedlecki, Tadeusz Pawlikowski i jego krakowscy aktorzy [Tadeusz Pawlikowski et ses acteurs cracoviens], Cracovie, Wydawnictwo Literackie, I97I, p. 77.

5. W. Ryszkowski, Wspomnienia [Souvenirs], manuscript, Biblioteka Narodowa w Warszawie, sgn. BN IV IOI89. 
avaient obtenu le droit à la retraite et pouvaient avec bonheur être indépendants, ne coupaient pas les liens avec la scène. De temps en temps, on leur organisait des festivités et des jubilés afin de célébrer leur belle carrière. Les retraités - selon une pratique mise en place au fil des ans - fréquentaient les cafés-théâtres, invitaient chez eux leurs jeunes collègues, s'immisçaient dans les coulisses du théâtre, participaient aux répétitions.

Un metteur en scène du théâtre de Poznań raconte ainsi la situation spécifique qui était celle d'avant-guerre :

Un jour, j'ai organisé une répétition technique à 8 heures du matin. On devait installer pour la première fois les éléments du décor. Je suis arrivé un peu avant. J'attendais les machinistes qui devaient arriver une demi-heure plus tard. La salle du théâtre était sombre, vide, seul un petit rayon de soleil traversant les rideaux éclairait la scène. Tout à coup, j'ai entendu des pas. Curieux de savoir qui se promenait au théâtre à cette heure-ci, je reculai dans la loge. Un homme aux épaules voûtées monta sur la scène. Il se tenait dans un mince faisceau de lumière, il ôta son chapeau melon, regarda en direction de la salle, se rapprocha de l'avant-scène et murmura longtemps quelque chose avec ferveur. Puis il se recouvrit la tête et sortit. Je courus rapidement en direction de la petite place devant le théâtre et je reconnus le visiteur. C'était l'acteur retraité Lochman, une ancienne vedette de Poznań. Apparemment, il faisait souvent cette petite excursion matinale, montait sur scène et se souvenait de l'époque révolue de sa gloire et de sa splendeur ${ }^{6}$.

La présence du Vieil Acteur dans Affranchissement est un témoignage de ses activités et de son inclination pour la scène.

À la fin du monologue, l'acteur accuse l'impuissance et la faiblesse du théâtre face aux obligations de la vie sociale. "Mon père était un héros, et moi je ne suis rien." L'ombre de son père résistant, ainsi qu'un puissant complexe lié à la disparition de l'État polonais et au statut de patrie déchue - qui caractérisait la culture polonaise du XIX ${ }^{\mathrm{e}}$ siècle, interfèrent dans le conflit entre l'art et l'engagement politique, l'action et la passivité. Chaque activité, y compris artistique, était alors évaluée à l'aune des valeurs dont la plus élevée était le sacrifice pour l'indépendance de la patrie. Sur ce plan-là aussi, la défaite du Vieil Acteur est flagrante, il n’a fait que «jouer» des tragédies, alors que son propre père avait, quant à lui, participé à un combat réel et tragique.

Chez Wyspiański, le Vieil Acteur fait l'expérience à un niveau dramatique élevé et intense de la coïncidence entre la sphère privée et la vie professionnelle. Il capitule sur tous les plans possibles. Il «est fini» en tant qu'artiste comme en tant qu'homme, la profession qu'il s'est choisie lui semble alors aussi triviale, éphémère et superficielle que la vie spirituelle de l'époque. Les grandes dimensions

6. J. Warnecki, Najdtuższy mój monolog [Mon plus long monologue], Varsovie, Czytelnik, I97I, p. 225. 
existentielles, artistiques, sociales et politiques suggérées par la phrase «Moi, je ne suis rien " pointent la condition de l'artiste : je n'existe pas comme personne, ni comme artiste, ni comme soldat ou militant, mon œuvre et mon être ne sont rien...

Parmi les nombreux portraits littéraires décrivant le Vieil Acteur de théâtre, la scène de L'Affranchissement est à la fois unique et représentative de la spécificité polonaise. Wyspiański a déterminé une certaine manière de regarder la mélancolie du vieux comédien, manière qui est difficile à rejeter et dont il est difficile de se «libérer». L'Affranchissement est un bel exemple de grande poésie et l'un des drames les plus intéressants du modernisme polonais. Le rôle du comédien usé fonctionne déjà au-delà du texte en question, il est un archétype littéraire circulant dans le monde du théâtre, associé autant à la réflexion sur l'art qu'au thème du passage et du vieillissement en général. Chaque acteur de la vieille génération s'y mesure quand il entre sur scène, même dans les pièces dont le sujet principal n'a rien à voir avec celui de la vieillesse. À l'ombre de la puissante imagination collective, se trouvent donc aussi ces doyens du théâtre polonais nés dans les années 1920 , et qui de temps en temps apparaissent dans les nouveaux spectacles, à côté de très jeunes interprètes. Ce sont eux que nous appelons ici les "vieux acteurs».

Le phénomène et le sentiment de la vieillesse sont relatifs, généralement définis sur la base d'un jugement subjectif. Leon Stępowski se sentait vieux à l'âge de cinquante ans, alors qu'un autre acteur polonais, Ludwik Solski (I855I954), ne faisait aucun cas du fait qu'il avait presque cent ans et jusqu'à la fin, il ne cessa de se produire sur scène et de s'adonner à tous les excès7. Nous nous intéresserons aux acteurs nés entre 1915 et 1928, âgés aujourd'hui de quatrevingts, quatre-vingt-dix ans ou plus. Certains ont célébré leur cinquantième, voire leur soixantième année de scène, ils ont fait leurs adieux au théâtre en grande pompe, avant d'y revenir, invités à jouer des rôles que de très jeunes metteurs en scène leur avaient préparés. Ces retours à la scène éveillent toujours une grande émotion, ils suscitent la curiosité, l'étonnement et la passion - et induisent des parallèles et des confrontations avec les souvenirs personnels du monologue du Vieil Acteur.

Quel rôle doit remplir un acteur octogénaire dans le théâtre polonais contemporain? Qui le pousse sur l'avant-scène, et pourquoi? La suite de notre analyse se détache de la réception du drame de Wyspiański et du personnage créé par

7. À la question sur la nature de la vieillesse, Solski a répondu à un acteur : «Tu vois, la vieillesse, c'est qu'autour de toi il y a de moins en moins d'amis et de compagnons qui ont ton âge» (J. Ciecierski, Mistrzowie i koledzy [Maîtres et amis], Varsovie, Wydawnictwa Artystyczne i Filmowe, 1978, p. 25). 
lui, pour aborder la problématique dans une perspective plus large : la présence des doyens de la scène polonaise dans les spectacles de cette dernière décennie.

\section{Le vieil acteur dans le théâtre polonais contemporain}

En 2009, au théâtre Jan Kochanowski d'Opole, s'est jouée une représentation de l'Odyssée mise en scène par Krzysztof Garbaczewski. Dans cet "essai scénique», Pénélope était jouée par trois actrices, dont la plus âgée était Zofia Bielewicz ${ }^{8}$. Ce n'était pas la figure la plus en vue de la pièce. La plupart des critiques ne mentionnèrent même pas sa présence, mais quand ils l'évoquèrent, ce fut pour mettre en cause cette Pénélope trop âgée et sclérosée, enfermée dans un rôle d'héroïne de second plan et ne faisant que répéter des truismes. L'actrice devait entre autres réciter le monologue de la mère d'Ulysse (Anticlée) lors de la rencontre à proximité de l'Hadès. La scène fit forte impression par sa performance vocale. Alors que les autres acteurs - avec une désinvolture délibérée effaçaient les mots et se contentaient d'un ton prosaïque et familier, Bielewicz récita son texte avec un sens de la syntaxe rhétorique et poétique héritée de sa formation artistique d'avant-guerre. Elle était probablement la seule personne sur scène qui n'avait besoin d'aucun micro pour renforcer sa voix naturelle. Sans aucun doute, le metteur en scène connaissait les capacités vocales de Zofia Bielewicz et voulait les utiliser pour produire un effet, à savoir surprendre l'auditoire par l'ancienne technique de déclamation à la fois délicate et sonore.

Un autre exemple, qui cette fois-ci révèle un usage perfide du potentiel vocal d'une actrice âgée, est illustré par la pièce intitulée La Guerre-Mère [Wojna matka], inspirée de Mère Courage et ses enfants et jouée au théâtre Stefan Jaracz de Łódź (2004). Le metteur en scène Remigiusz Brzyk confia le rôle principal à Irena Burawska'. La condition physique de l'actrice produisit un décalage net entre son interprétation du rôle et les intentions de Brecht. Elle devint une "Grand-Mère Courage», une "vieille femme frêle qui certes possède une grande expérience de la vie, mais qui a perdu toute son énergie et beaucoup d'émotion ${ }^{10}{ }^{1}$. La première observation liée à la présence du Vieil Acteur sur la scène contemporaine touche

8. Z. Bielewicz est née en 1928 à Varsovie. Pendant la guerre, elle a joué dans un théâtre clandestin et, après I945, dans différents théâtres d'Opole, Olsztyn, Łódź et Lublin. Elle s'est ensuite principalement produite au théâtre Jan Kochanowski à Opole, où, en l'an 2000, elle a fêté ses cinquante ans de scène.

9. I. Burawska-Kesling (1922-2007), diplômée de l'école de théâtre de Varsovie, après une courte période de travail à Lublin et Opole, joua principalement dans les théâtres de Łódź et depuis 1966 au théâtre Stefan Jaracz. Actrice épisodique, elle joua dans des dizaines de films. Elle est morte à Skolimów, dans la maison de retraite des artistes.

Io. B. Guczalska, «Babcia Courage» [Grand-Mère Courage], Didaskalia, nº 63, 2004, p. 43. 
donc le métier lui-même, et plus strictement la tradition de la diction. En invitant sur les planches les doyens du théâtre, les metteurs en scène introduisent un effet d'éloignement culturel, font appel à une forme de la langue polonaise presque totalement perdue : en témoignent la représentative consonne coronale $ł$, la sonore h, la diction claire, stylée et mélodique, ainsi que la culture orale au sens large. On ne parle plus comme ça. L'acteur fonctionne en quelque sorte comme une relique de l'éloquence et du style de jeu, la survivance de l'ancien ordre intellectuel et esthétique. La noblesse et le raffinement dominent, mais un tel style peut aussi se révéler stérile et impuissant dans son costume anachronique du théâtre ancien. Il inspire l'admiration et la nostalgie, mais laisse aussi une fâcheuse impression de discontinuité avec les mœurs contemporaines.

Outre leur usage archaïque de la langue, les vieux acteurs attirent l'attention par l'aura scénique qui se produit autour d'eux. Ils peuvent n'appliquer aucune gestuelle propre au théâtre, se contenter de se tenir immobile et silencieux - et malgré tout éveiller une profonde émotion. Quelle que soit la manière dont ils interprètent leur rôle au point de vue purement artistique, les spectateurs évaluent leur prestation comme une confession directe, comme une représentation biographique de l'acteur. Danuta Szaflarska est un bon exemple pour illustrer cette idée. Née en I9I5, elle a été choisie par Grzegorz Jarzyna pour le rôle de la Vieille Prostrée de la pièce Vive le feu! de Dorota Masłowska, jouée au Teatr Rozmaitości (TR Warszawa) à Varsovie en 2009. Le jour de la première, l'actrice avait quatre-vingt-quatorze ans. En février 2015 , elle a fêté son centième anniversaire et joue encore (d'ailleurs le spectacle figurait toujours en avril 20I5 au répertoire du théâtre $)^{\text {II }}$. Dans les interviews, Szaflarska a parlé à plusieurs reprises de ses expériences de la guerre (elle était agent de liaison dans la Résistance polonaise) et de sa carrière d'avant et d'après-guerre. Mais dans ces entretiens, elle fait une distinction nette entre son travail au théâtre et son autobiographie, en mettant l'accent avant tout sur son métier d'actrice : «Je suis extrêmement contente d'avoir l'occasion de travailler." "Je joue chez Grzegorz Jarzyna! Et en plus, comme si cela n'était pas assez, je travaille sous contrat! Vous rendez-vous compte? ${ }^{12}$ »

II. D. Szaflarska fit ses débuts sur scène à Varsovie en 1939. Pendant l'occupation elle joua dans le théâtre clandestin. Elle participa à l'Insurrection de Varsovie (1944). Après la guerre, elle travailla à Cracovie, Łódź et surtout à Varsovie. Son rôle dans le premier film polonais d'après-guerre, Zakazane piosenki (Les chants interdits, 1946), lui ouvrit une longue carrière cinématographique. En 2008, elle fut reconnue meilleure actrice du cinéma polonais du $\mathrm{XX}^{\mathrm{e}}$ siècle. Récemment, en 20I4, elle a tenu le rôle de la grand-mère dans la version cinématographique de l'œuvre Vive le feu!

I2. J. Dąbrowska, «Danuta Szaflarska: Myślę do przodu» [Danuta Szaflarska : Je pense au futur], Tygodnik Powszechny, n ${ }^{\circ}$ I5, 2012. 
Pourtant le public et les critiques n'ont pas vu dans la Vieille Prostrée un rôle incarné par l'actrice, mais plutôt une extension de la personne Danuta Szaflarska - cette femme qui avait survécu à la Seconde Guerre mondiale et qui revenait sur son passé, subjuguant tout le monde avec sa personnalité charmante et son humanité rayonnante : «Grzegorz Jarzyna a choisi pour jouer le rôle de La Vieille Prostrée, la merveilleuse Danuta Szaflarska, une grand-mère-chaleureuse, une grand-mère-pleine-de-sagesse, une grand-mère-qui-impose-le-respect, une grand-mère dont chacun voudrait être le petit-fils. Une grand-mère dont le seul regard exclut toute interprétation grotesque ${ }^{13}$.» Szaflarska se distinguait des autres rôles, joués comme des créatures repoussantes, car le public interprétait sa présence sur la scène comme autoréférentielle. Il est intéressant dans ce contexte de replacer le fragment du monologue de Vieil Acteur de Wyspiański touchant à l'anti-héroïsme. La Vieille Prostrée jouée par Szaflarska non seulement n'était pas grotesque, mais réfutait aussi le sentiment de néant et d'inutilité du Vieil Acteur. Le public voyait une personne qui avait pris part à la guerre, qui avait été témoin de l'histoire, qui avait participé aux plus importants événements $\mathrm{du} \mathrm{xx}^{\mathrm{e}}$ siècle. C'étaient cette fois-ci les spectateurs qui pouvaient dire d'euxmêmes : "Moi, je ne suis rien."

En contrepartie de ce regard du public qui englobe le rôle et la personnalité privée de l'actrice, il y a la peur pour sa santé et sa vie. Regarder un vieil acteur suscite chez le spectateur la crainte de sa disparition. L'inquiétude individuelle et instinctive précède ici toute réflexion générale et théorique sur la mort. Ainsi, le «drame scénique» devient un «drame existentiel » dans la réalisation des pièces de Ionesco, Les Chaises et Le roi se meurt, au Théâtre polonais de Wrocław. C'est Igor Przegrodzki qui a joué dans ces deux pièces, un acteur qui depuis plus de quatre décennies s'est dévoué à la scène théâtrale de Wrocław ${ }^{14}$. En 1994, il a joué le Vieux (mise en scène de Maciej Englert), et en 1996 Bérenger (sous la direction de Jerzy Grzegorzewski). Dans ces rôles-là comme dans les rôles suivants de Przegrodzki (Kazimierz Kamiński dans Hamlet de Wyspiański et le Comédien dans Lui. Le Deuxième Retour d'Ulysse $\left.e^{\mathrm{IS}}\right)$, le thème de la mort à venir est lié au thème théâtral du dernier rôle impossible à jouer, de la dernière représentation. Cette interprétation est présente dans la trame même des drames d'Ionesco et de Wyspiański. Il est impossible de ne pas remarquer

13. J. Sieradzki, «Zbombardowani» [Bombardés], Odra, nº 7-8, 2009.

I4. I. Przegrodzki (1926-2009) s'est formé au théâtre dans une école clandestine de Vilnius. Il fit ses débuts en I945, travailla à Toruń, Olsztyn, Poznań et surtout, de I949 à l'an 2000, il fut lié à la scène de Wrocław. Excellent acteur de théâtre, de cinéma et de télévision, il fut aussi directeur du Théâtre polonais de Wrocław, pédagogue et réalisateur.

I5. Les deux pièces ont été mises en scène par J. Grzegorzewski au Théâtre national de Varsovie en 2003 et 2005 . 
que la lecture existentielle de ces textes est rendue plus forte grâce à l'émotion née de la condition, de l'âge et de la fragilité de l'interprète. Contrairement à cette attitude du public, les vieux acteurs n'évoquent généralement qu'avec discrétion et réticence la "fin de vie». Cela n'est en soi guère surprenant. «La mort - c'est personnel», tranche Szaflarska dans une interview. Jerzy Nowak $^{16}$, pour protester contre le phénomène visant à rendre publique la vie privée de l'acteur, a organisé une provocation qui a fait beaucoup de bruit. Il a annoncé en 2005 qu'il était mortellement malade et que pour cette raison il avait l'intention de tourner avec Marcin Koszałka un film sur ses derniers moments et sur son enterrement (symbolique, parce que son corps devait en réalité être donné à la science). Il a déclaré aux journalistes :

Et si je vous disais que je fais tout pour la gloire et pour ma carrière? J'ai joué des dizaines de seconds rôles, j’ai gagné enfin le droit de jouer le héros [...] En brisant une certaine limite, je veux laisser mon empreinte. En tant qu'acteur, j'ai pendant toute ma vie appris à faire semblant. Je veux enfin être moi-même. Aller aussi loin que possible. Jouer moi-même. Jusqu'à la fin ${ }^{17}$.

Tout cela s'est évidemment avéré être une blague : «Maintenant, je vais jouer un vieil acteur cynique qui, pour la gloire, dévoile son agonie devant la caméra $^{18}$." Nowak ne souffrait d'aucune maladie mortelle et il n'est pas mort pendant le tournage du film (qui a été effectivement tourné, mais sur un sujet légèrement différent : L'Existence dirigé par M. Koszałka, 2007). Par contre, l'acteur a réussi à attirer l'attention sur la dimension médiatique de sa mort éventuelle et a marqué ses distances avec une telle éventualité de médiatisation. Dans ses interviews ultérieures, il n'a cessé d'affirmer que ce qui concernait sa mort, son testament et son enterrement relevait du domaine privé.

Il résulte de ce qui a été dit que les doyens du théâtre sont condamnés à dévoiler leurs conditions de vie : leurs conditions de personnes âgées, affaiblies, tombant dans l'oubli ou devenant les légendes de leurs succès anciens. Ce n'est que grâce à ces confessions qu'ils rencontrent encore la reconnaissance, la

I6. J. Nowak (1923-20I3), diplômé de l'école dramatique de Cracovie, a toute sa vie été lié à la scène de cette ville. Il est intéressant de remarquer qu'il a interprété des rôles de personnages âgés depuis les débuts de sa carrière. En I957, alors trentenaire, il a joué aux côtés de H. Gallowa le rôle du Vieux dans la pièce Les Chaises, mise en scène par J. Grotowski et A. Mianowska. Dans les années 1950, il était aussi acteur du théâtre Cricot 2 de T. Kantor. Il a joué dans des dizaines de films et interprétait volontiers le rôle de personnages juifs.

I7. Ł. Maciejewski, "Jerzy Nowak: Na koniec chcę być sobą» [Jerzy Nowak : Avant de mourir, je veux être moi-même], Gazeta Wyborcza, nº 193, 2005.

I8. W. Krupiński, «Istnienie, nie śmierć» [L’existence, pas la mort], Dziennik Polski, 22 VIII 2005, <http://www.dziennikpolski24.pl/> [l'accès : 3 XI 20I4]. 
sympathie et la tendresse du public - un écho positif, parfois même cordial ${ }^{19}$. Cette problématique semble être toutefois beaucoup plus complexe.

Il est intéressant de comparer le spectacle de Jarzyna Vive le feu! que nous avons évoqué ci-dessus avec la représentation d'Alicja [Alice], de Paul Miśkiewicz, en 2008 au Théâtre dramatique de Varsovie. Le rôle d'Alice "adulte» était tenu par Barbara Krafftówna ${ }^{20}$. Quelles que soient les différences entre le conte onirique et surréaliste de Carroll et la pièce sarcastique et grotesque de Masłowska, les deux représentations se rejoignent par le traitement du motif du sosie. Le personnage d'une vieille femme côtoie celui d'une jeune fille : deux Alice se sont rencontrées dans la pièce de Miśkiewicz alors que chez Jarzyna interviennent la Vieille Prostrée et la Jeune Fille en Métal. La Vieille Femme était habillée comme son jeune sosie : toutes deux portaient une robe et elles avaient les cheveux tressés, si bien qu'elles paraissaient être jumelles, elles pouvaient se mirer l'une dans l'autre, chacune étant à la fois la vieillesse et la jeunesse. Alice d'un côté et de l'autre côté du miroir, la fille d'un côté et de l'autre côté de la guerre. Les deux personnages exprimaient la durée de la vie, illustraient un trait typique de la vieillesse, celui de pouvoir revenir en arrière, d'entamer un voyage dans la mémoire, vers des époques de plus en plus éloignées, aux sources même de l'expérience personnelle. Cela aurait pu se transformer en une nostalgique et sentimentale reconnaissance des anciennes saveurs, expériences et aventures, mais en réalité ce sont les propriétés étonnantes du vieux «médium» qui se sont révélées. La mimique, la grâce, l'imprévisibilité spécifique du jeu de Danuta Szaflarska et de Barbara Krafftówna ont fait que, paradoxalement, les deux actrices sont devenues sur scène... des jeunes filles.

Barbara Krafftówna ne joue pas le personnage d'Alice de Carroll, mais elle l'est tout simplement : une petite fille désarmante aux longs cheveux roux, au sourire malicieux et à la voix timide. Une jeune fille résolue âgée de onze ans et de centaines de mois ${ }^{21}$.

19. Ces émotions ont suscité deux films récents, qui se sont inspirés de cette problématique : Pora umierać [Il est temps de mourir] de D. Kędzierzawska (2007) et Jeszcze nie wieczór [Le soir n'est pas encore arrivé] de J. Bławut (2009); ce film a d'ailleurs été tourné dans la maison de retraite des acteurs de Skolimów.

20. B. Krafftówna, née en I928, débuta ses études dramatiques à Cracovie pendant l'Occupation, puis joua à Gdynia, Łódź, Varsovie, principalement dans le répertoire de l'avant-garde (les pièces de Witkiewicz) et sur la scène des cabarets (Studencki Teatr Satyryków), ainsi qu'au cinéma et à la télévision. Dans les années I980, exilée en Amérique, elle a joué au Théâtre Polonais. En 20Io, elle a célébré à Varsovie le soixantième anniversaire de sa vie d'actrice. Elle porte toujours son nom de famille en ajoutant le suffixe - ówna qui indique qu'elle est une femme célibataire. C'est une forme onomastique désuète aujourd'hui.

2I. A. R. Burzyńska, «Trudna sztuka kopiowania snów» [L'art difficile de copier les rêves], Tygodnik Powszechny, n 43, 2008. 
Ce n'est plus là une représentation de la vieillesse, mais la révélation de la maturité et de la fascinante personnalité d'une actrice qui a annexé les différentes étapes de la vie, qui a absorbé de nombreux rôles et incarnations, qui est capable de les faire apparaître et de leur donner délibérément la forme qu'elle veut. Les deux actrices étaient étonnamment métamorphiques, flexibles, prêtes à l'auto-ironie tout autant qu'à une volte-face stylistique pleine de virtuosité. De façon assez inattendue, Alice-Krafftówna a enchanté le public avec la chanson de Kurt Weill, Speak Low. Quant à la très distinguée Szaflarska, son style raffiné a été brisé par le ton vulgaire et sarcastique dans lequel se superposaient des expressions juvéniles aux "carafes», aux "petits fours» et aux épithètes surannées ${ }^{22}$.

La prestation des deux actrices est fractionnée en deux pôles : d'un côté la jeunesse, la vitalité, l'agilité, la grâce, la subtilité lyrique et de l'autre la laideur, l'étrangeté, la difformité. Le Vieil Acteur est celui qui peut tout jouer, parce qu'il a tout connu. L'étendue de son expérience et de ses compétences est si grande qu'il se situe au-delà des catégories d'âge ou de sexe. Le corps et la voix, ciselés pendant de longues années de métier, ressemblent à la copie parfaite d'un Stradivarius.

Il y a toutefois un pendant sombre de ces métamorphoses. La présence scénique du vieil acteur met à nu le mystère du corps, compris à la fois comme la matière plastique du rôle et comme une matière sujette à la fugacité, inscrite dans un processus de destruction inéluctable. Dans le monde du théâtre polonais, l'actrice Halina Mikołajska (1925-1989) illustre parfaitement cette idée. Très tôt, elle a été confrontée au défi de jouer une "vieille centenaire» :

La profession d'acteur relevant du domaine public, il en découle que sa date de naissance est généralement connue. Le succès d'une jeune fille en plein épanouissement n'est pas que le fruit de sa vive sensibilité et de sa sensualité, de la spontanéité de son esprit ou de son absorptivité psychique, mais il est également dû à son charme, à la finesse de son cou, à sa belle tête fixée sur une colonne vertébrale saine et sans rhumatisme, à l'éclat de ses yeux, pas encore assombri par la chute sur les rares derniers cils des paupières flasques et ridées. Ce succès si souvent reconnu publiquement... Quel danger! Quel risque de confondre entre les moyens et le but même et, enfin, quelle dégradation de ses capacités. En effet, afin de continuer à être créateur, afin de continuer à être en mesure de dire la vérité, il faut encore être capable de puiser dans son authentique, sincère et quasi physiologique "vie privée». Il est parfois nécessaire de dévoiler son cou qui n'a plus rien de la splendeur d'un cygne, mais qui ressemble plutôt à la pomme d'Adam ratatinée d'une poule, et de

22. Dans le film Pora umierać (Il est temps de mourir, dirigé par D. Kędzierzawska, 2007) Szaflarska a ici aussi montré son tempérament et sa "griffe de lion». Le film débute avec une fulgurante réplique - «Je t’emmerde!» : c’est ainsi que la grand-mère désœuvrée répond dans une clinique à un médecin qui lui manque de politesse. 
regarder le public dans les yeux avec ses prunelles rétrécies qui n'ont plus rien de leur ancien éclat triomphal, pour lui dire tout aussi clairement cet autre aspect - le même ou non, en tout cas nouvellement découvert grâce au temps - de la vérité. C'est comme si l'on usait pour la dire d'un instrument différent. En général, il faut dès le début être capable non seulement d'éblouir, mais aussi d'effrayer ou même d'écœurer, comme dans les peintures de Jérôme Bosch ou dans le Bestiaire de Jan Lebenstein. Il peut arriver que seules cette peur et cette conscience exacerbée du passage puissent éveiller chez le destinataire un amour d'un genre plus élevé $[\ldots]^{23}$.

Le Vieil Acteur, c'est une tête qui ne se souvient pas, un corps flétri, tremblotant et "dévasté», une féminité dépourvue de sensualité, un autre qui naît en chacun à la fin de sa vie. Le Vieil Acteur, assimilé aux peintures de Bosch! Ainsi, l'acteur n'est pas seulement une idole, dont la vie fait frémir les spectateurs fidèles, mais aussi un Étranger, un Monstre, qui effraie le public avec sa propre angoisse et qui le force à chercher une autre beauté, située au-delà des sens, ainsi qu'une autre sensibilité, cachée sous la peau du corps.

Les apparitions sur scène des acteurs seniors ont plusieurs points communs : une formidable technique vocale et physique, une tendance à être perçus du public comme personnes plutôt que comme personnages, et en même temps une capacité de métamorphoses dans le jeu (avec la coexistence dans un corps de différentes entités : jeune-vieille femme, femme-homme, humain-nonhumain). Au théâtre, les doyens entraînent invariablement avec eux le problème universel de la condition humaine. "Le thème du vieil acteur est un thème porteur, car en lui il y a tout le drame de la vie. Il y a là à vrai dire tout ce que vit chaque être humain, mais sous une forme multiple ${ }^{24}$ ».

Parmi les sujets évoqués dans le monologue du Vieil Acteur d'Affranchissement, le plus actuel s'est donc révélé être le thème existentiel. La formule ironique "Moi, je ne suis rien» a pris une dimension métaphysique inattendue. Les Vieux Acteurs sont des modèles uniques d'un monde qui n'est plus : la Pologne de jadis, l'ancienne école de théâtre, une autre société. Ils renvoient à un sens et à un contexte qui pour la plupart des spectateurs sont tout simplement inaccessibles. Le Vieil Acteur crée des signes qui n’ont plus de référent, qui

23. H. Mikołajska, «Aktor jako osoba prywatna i aktor jako osoba publiczna» [L'acteur comme personne privée et comme personne publique], dans: Świadomość teatru. Polska myśl teatralna drugiej potowy XX wieku [La conscience du théâtre. La pensée polonaise du théâtre pendant la seconde partie du XX siècle], éd. W. Dudzik, Varsovie, Wydawnictwo Naukowe PWN, 2007, p. 4 II.

24. Ce sont les mots de l'acteur Tadeusz Łomnicki, cité par B. Guczalska, Aktorstwo polskie. Generacje [Les acteurs professionnels polonais. Générations], Cracovie, Państwowa Wyższa Szkoła Teatralna im. Ludwika Solskiego, 20I4, p. I36. 
frappent dans le vide. On peut qualifier cette faculté de «spectrale». Prenons deux exemples, dans le théâtre et le cinéma, pour illustrer cette idée.

Jerzy Nowak, que nous avons déjà mentionné ci-dessus, avait dans son répertoire un type spécifique de rôles que nous ne pouvons comprendre si l'on fait abstraction de la biographie de l'acteur. Nowak est né avant la guerre, dans les environs de Lvov (son père était staroste de la ville de Bohorodczany). Pendant l'Occupation, encore adolescent, il a servi dans un groupe de résistants. Dès sa prime jeunesse, comme il le dit lui-même, il a appris le "dialecte juif ${ }^{25}$ ». Le rôle des personnages juifs devint sa spécialité, à la fois dans les films ( $\mathrm{La}$ Terre de la grande promesse d'Andrzej Wajda, Le Sanatorium au croque-mort de Wojciech Jerzy Has, La Liste de Schindler de Steven Spielberg) et au théâtre (Les Juges avec le metteur en scène Konrad Swinarski). En 1994, il a joué pour la première fois le rôle principal dans la représentation Ja jestem Żyd $z$ Wesela [Moi, je suis le Juif des Noces], une adaptation du récit de Roman Brandstaetter (au Vieux Théâtre de Cracovie, sous la direction de Tadeusz Malak). Il a joué cette pièce 633 fois, pratiquement jusqu’à sa mort. N'ayant pas de racines juives, il était pourtant le principal représentant de l'individu juif grâce à ses traits et à son expérience d'avant-guerre. Il a recréé la silhouette du Juif, qui avait complètement disparu du paysage polonais, mais, comme on l'a dit, en la protégeant contre le stéréotype, le conventionnel, la caricature. L'intuition créatrice de l'acteur a été guidée par le fait qu'il gardait en mémoire le prototype que le public, lui, n'avait plus la possibilité de vérifier, c'est-àdire de trouver un point de référence dans le monde qu'il connaissait. Il était l'apparition du dernier témoin visible, en quelque sorte une créature spectrale, une incarnation spirituelle. Ce n'est pas seulement à cause de la spécificité des rôles que Jerzy Nowak a joués mais, de façon plus générale, en raison de sa formation professionnelle et psychologique que son ami Jerzy Trela l'a appelé «le dernier des Mohicans ${ }^{26}$ ».

Le deuxième exemple de «faculté spectrale» du Vieil Acteur vient du film d'Andrzej Wajda, La Terre de la grande promesse. Le film est l'adaptation d'un roman qui s'inscrit dans le monde d'une ville polonaise (Łódź) au XIX siècle. Władysław Reymont, l'auteur du roman, a commencé à rédiger son épopée de Łódź en I896, deux ans avant que naisse, dans cette ville industrielle et dans une famille juive, l'un des plus grands poètes polonais du $\mathrm{xx}^{\mathrm{e}}$ siècle - Julian Tuwim. Dans le roman de Reymont, le poète Tuwim - fondateur du

25. Dans le livre de souvenirs Książka o mitości (Livre sur l'amour, Cracovie-Budapest, Austeria, 2009), qui décrit de fugaces et parfois poignantes impressions, J. Nowak et M. AndruszkiewiczNowak évoquent la silhouette de quelques camarades d'école, dont David Zukerman et Aron Brossa.

26. J. Nowak, M. Andruszkiewicz-Nowak, Książka o mitości, ouvr. cité., p. I28. 
groupe Skamander et l'auteur des Fleurs polonaises [Kwiaty polskie], n'intervient évidemment pas. Mais dans le film, il apparaît comme un personnage un peu fantastique incarné par un acteur de l'ancienne génération, Włodzimierz Boruński (I906-I988). Appelé le «maître des épisodes», il a joué à l'écran des personnages secondaires, alors qu'au théâtre il a davantage été associé à la "muse légère». Pour La Terre de la grande promesse, il aurait été engagé à cause de sa ressemblance physique avec Julian Tuwim, avec lequel il avait d'ailleurs des liens de parenté. Dans le film, Boruński a joué un rôle épisodique tout fictif, mais il a également joué son cousin poète. Andrzej Wajda a causé, bon gré mal gré, une série d'associations étranges. La physionomie de Boruński avait une ressemblance frappante avec les photos d'après guerre de Tuwim. C'était le même visage ascétique, aux traits fins et aux yeux perçants, que celui de Tuwim quand il regardait l'objectif de l'appareil photographique sur une image qui date de la fin de la guerre. En 1975, le vieux Włodzimierz Boruński a donc joué, vingt ans après sa mort, le poète et Juif polonais Julian Tuwim, quand il déambulait dans la ville de Łódź à la fin du Xixe siècle, avec son visage émacié de 1945. Piotr Matywiecki écrit à ce sujet :

Dans La Terre de la grande promesse, Boruński dirigé par Wajda «représente» Tuwim, il est le vivant représentant du poète défunt, marchant comme un fantôme dans la ville de sa jeunesse. En même temps, il incarne l'esprit d'un Juif de Łódź - le seul parmi les Juifs à avoir été stylisé comme une personne "de chair et de sang". À travers ce visage spectral pénètrent d'autres personnages du film comme y pénètre le visage du public - ce visage spectral change les uns et les autres en fantôme. Le visage de Boruński est le visage de Tuwim après l'extermination ${ }^{27}$.

Le visage de Boruński scelle le secret le plus profondément caché du rôle du Vieil Acteur : son appartenance à un autre temps et à un autre monde. Il faut noter néanmoins une différence entre le cinéma et le théâtre. Le cinéma est par principe spectral, il se base sur l'empreinte humaine laissée sur la pellicule, par opposition au théâtre, où il y a coexistence physique entre le spectateur et l'acteur. Tandis qu'au cinéma nous frayons avec une image rémanente de l'acteur, au théâtre l'impression de communion avec une personne qui n'est plus qu'à moitié présente, est plus complexe et indirecte. Le caractère spectral du vieil acteur tient à l'effacement de la lisibilité des gestes, des mimiques, de la voix et du visage, c'est une ouverture à une dimension qui nous est totalement inaccessible. Et fascinante.

27. P. Matywiecki, Twarz Tuwima [Le visage de Tuwim], Varsovie, Wydawnictwo WAB, 2007, p. 66-67. 